\title{
Divisor Graphs that are Complements of Bipartite Graphs
}

\section{Ahmad $\mathrm{M}$ and $\mathrm{Al}$-Ezeh $\mathrm{H}^{*}$}

Department of Mathematics, Faculty of Science, University of Jordan, Amman, Jordan

\begin{abstract}
In this paper, we will study some bipartite graphs whose complements are divisor graphs like paths and caterpillars, counterexample for a tree whose complement is not divisor will be presented and powers of some types of trees that have their complements divisor graphs will be classified.
\end{abstract}

Keywords: Divisor graph; Complement of a graph; Bipartite graph; Path; Caterpillar power of a graph and tree

\section{Introduction}

A finite graph $G$ is called divisor graph if there is a finite set of positive integers $\left\{x_{1}, x_{2}, \ldots, x_{n}\right\}$ such that $V(G)=\left\{x_{1}, x_{2}, \ldots, x_{n}\right\}$ and $\left\{x_{i}, x_{j}\right\} \in$ $E(G)$ if $x_{i} / x_{j}$ or $x_{j} / x_{i}$.

It is known that bipartite graphs are divisor graphs but what about their complements? Are they all divisor graphs? The answer is no. we will study some special cases of bipartite graphs that have their complements divisor graphs like paths and caterpillars, while other types like some trees are not.

We know that every tree is a divisor graph [1]. The question that arises here, is the complement of a tree is also a divisor graph?

Characterization of block graphs that are divisor graphs are given [1].

Definitions in terms of transmitter, receiver and transitive vertices of a divisor orientation of a graph $\mathrm{G}$ are given [2].

The characterization of powers of paths and powers of cycles which are divisor graphs was given [2-4]. While, a characterization of nontrivial connected divisor graphs in terms of the upper orientable hull number was obtained [5].

It was shown that no divisor graph contains an induced odd cycle of length greater than 3 . Also, it was proved that every induced subgraph of a divisor graph is a divisor graph [6-8].

Complete graphs, bipartite graphs, complete multipartite graphs, and joins of divisor graphs are divisor graphs.

The length of a longest path [9-15]. While divisor graphs with triangles [16], where a forbidden subgraph characterization for all divisor graphs containing at most 3 triangles was obtained.

\section{Lemma}

The complement of a path is a divisor graph (Figure 1).

Proof

Consider the path $P_{n=}\left\{v_{1}, v_{2}, \ldots, v_{n}\right\}$ with $\left\{v_{k}, v_{k+1}\right\} \in E\left(P_{n}\right)$ for $k=1,2, \ldots, n-1$. Let

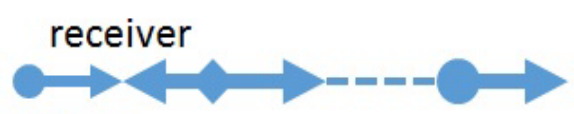

\section{transmitter}

Figure 1: A complement divisor graph. $\left\{p_{1}, p_{2}, \ldots, p_{n}\right\}$ be a set of distinct prime numbers. Label $v_{1}$ by $p_{1}$,

$v_{2}$ by $p_{2}$ and

$v_{k}$ by $p_{1} p_{2} p_{3} p_{4} \ldots p_{k-2} p_{k}, k=1,2, \ldots, n$.

This recurrence relation gives an orientation for $\bar{P}_{n}$, the complement of $P_{n}$ (Figure 2).

\section{Example}

$C_{n}$ : For odd $n \geq 5$ is not a divisor graph.

Example

$\mathrm{K}_{\mathrm{n}}$ : is a divisor graph (any complete graph is a divisor graph).

Example

Every bipartite graph is a divisor graph.

\section{Lemma}

The complement of a caterpillar is a divisor graph.

\section{Proof}

$P_{n=}\left\{v_{1}, v_{2}, \ldots, v_{n}\right\}$ with $\left\{v_{k}, v_{k+1}\right\} \in E\left(P_{n}\right)$ for $k=1,2, \ldots, n-1$.

Assume that the vertex $v_{k}$ is adjacent to the vertices $\left\{v_{k 1}, v_{k 2}, \ldots, v_{k j}\right\}$ that are not adjacent to any other vertex in $P_{n}$. Then the new graph $G$ is a caterpillar with $n+j$ vertices.

Label $v_{1}$ by $p_{1}, v_{2}$ by $p_{2}$ and $v_{k}$ by $p_{1} p_{2} p_{3} \ldots p_{k-2} p_{k}, k=1,2, \ldots, n$.

Label $v_{k} 1$ by $p_{1} p_{2} p_{3} \ldots p_{k-2} p_{k-1}$, $v_{k 2}$ by $v_{k 2}$ by $p_{1} p_{2} p_{3} p_{4} p_{k-2} p_{k-1} p_{k}, v_{k 3}$ by $p_{1} p_{2} p_{3} \ldots p_{k-2} p_{k-1} p_{k} p_{k+1}$,

$\ldots$ and $v_{k j}$ by $p_{1} p_{2} p_{3} \ldots p_{k-2} p_{k-1} p_{k} p_{k+1} \ldots p_{k+j-2}$.

And label the rest as follows:

$v_{k+1}$ by $p_{1} p_{2} p_{3} \ldots p_{k-2} p_{k-1} p_{k+1} \ldots p_{k+j-1}$

$v_{k+2}$ by $p_{1} p_{2} p_{3} \ldots p_{k-1} p_{k} p_{k+2} \ldots p_{k+j-1} p_{k+j}$

And so on till

*Corresponding author: Al-Ezeh $\mathrm{H}$, Department of Mathematics, Faculty of Science, University of Jordan, Amman, Jordan, Tel: +962 6535 5000; E-mail: morad.ahmad@ju.edu.jo

Received October 23, 2017; Accepted March 30, 2018; Published April 09, 2018

Citation: Ahmad M, Al-Ezeh H (2018) Divisor Graphs that are Complements of Bipartite Graphs. J Appl Computat Math 7: 400. doi: 10.4172/2168-9679.1000400

Copyright: @ 2018 Ahmad M, et al. This is an open-access article distributed under the terms of the Creative Commons Attribution License, which permits unrestricted use, distribution, and reproduction in any medium, provided the original author and source are credited. 


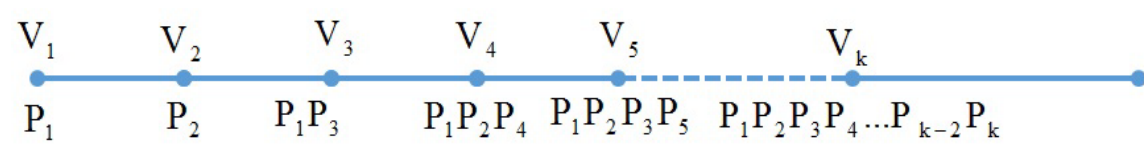

Figure 2: Recurrence Relation orientation for $\bar{P}_{n}$.
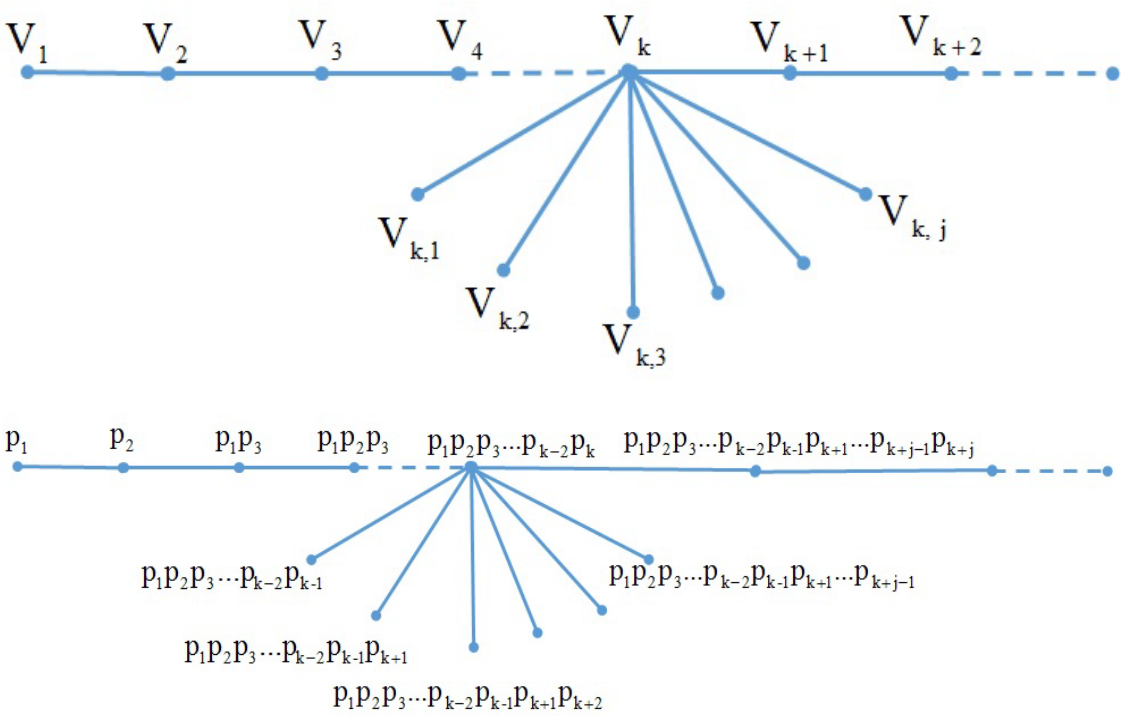

Figure 3: Recurrence orientation for $\bar{G}$.

$v_{n}$ by $p_{1} p_{2} p_{3} \ldots p_{k-1} p_{k} p_{k+1} \ldots p_{k+j-1} p_{k+j} p_{n-2} p_{n}$.

This recurrence relation gives an orientation for $\bar{G}$, the complement of $\mathrm{G}$ (Figure 3).

\section{Example}

Not all the trees are divisor graphs, for this purpose, consider the following tree:

This is not a divisor graph (Figure 4).

\section{Theorem}

The complement of a tree is a divisor graph only if the tree is a caterpillar.

\section{Proof}

Obvious from the above results.

The question that arises now is, are the complements of the powers of the graphs studied above also divisor graphs? Lemma.

The answer is yes, which we will prove it using the following

\section{Lemma}

The complement of the power graph of a path is a divisor graph. (For any power less than the degree of a path).

\section{Proof}

Consider the path $P_{n=}\left\{v_{1}, v_{2}, \ldots, v_{n}\right\}$ with $\left\{v_{k}, v_{k+1}\right\} \in E\left(P_{n}\right)$ for $k=1,2, \ldots, n-1$. Label $v_{1}$ by

$p_{1}$,

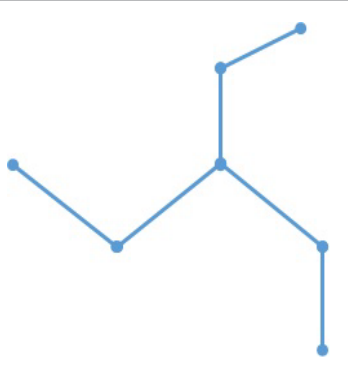

Figure 4: Trees are not divisor graphs.

$v_{2}$ by $p_{1}, \ldots$ and

$v_{j}$ by $p_{j}$. Then label $v_{j+k}$ by $p_{1} p_{2} \ldots p_{k} p_{j+k}$, Finally, label $v_{n}$ by $p_{1} p_{2} \ldots p_{n-j^{*}}$

This recurrence relation gives an orientation for $\left(\bar{P}_{n}\right)^{j}$, the complement of $\left(P_{n}\right)^{j}$.

\section{Lemma}

The complement of the power graph of a caterpillar is a divisor graph.

\section{Theorem}

The complement of the power graph of a tree is a divisor graph only if the tree is a caterpillar.

\section{Proof}

Obvious from the above results. 
Citation: Ahmad M, Al-Ezeh H (2018) Divisor Graphs that are Complements of Bipartite Graphs. J Appl Computat Math 7: 400. doi: 10.4172/21689679.1000400

Page 3 of 3

\section{References}

1. Al-Addasi S, AbuGhneim OA, Al-Ezeh H (2012) Further new properties of divisor graphs. JCMCC 81: 261-272.

2. Al-Addasi S, AbuGhneim OA, Al-Ezeh H (2010) Divisor orientations of powers of paths and powers of cycles. Ars Combin 94: 371-380.

3. Al-Addasi S, AbuGhneim OA, Al-Ezeh H (2010) Characterizing powers of cycles that are divisor graphs. Ars Combin 97: 447-451.

4. Buckley F, Harary F (1990) Distance in graphs. Addison-Wisley Publishing, p: 335.

5. Chartrand G, Fink JF, Zhang P (2003) The hull number of an oriented graph Int J Math Sci 36: 2265-2275.

6. Chartrand G, Muntean R, Saenpholphat V, Zhang P (2001) Which graphs are divisor graphs? Congr. Numer 151: 189-200.

7. Chudnovsky M, Robertson N, Seymour P, Thomas R (2006) The strong perfect graph theorem. Ann Math 1: 51-229.

8. Chvatal V, Sbihi N (1988) Recognizing claw-free perfect graphs. J Combin Theory Ser B 44: 154-176.
9. Edros P, Freud R, Hegyvari N (1983) Arithmetical properties of permutations of integers. Acta Math Hungar 41: 169-176.

10. Gera R, Saenpholphat V, Zhang P (2003) Divisor graphs with triangles. Congr Numer 161: 19-32.

11. Gross JL, Yellen J, Zhang P (2004) Handbook of Graph Theory. CRC Press LLC, Boca Raton pp: 642-660.

12. Harary F (1963) A characterization of block-graphs. Canad Math Bull 6: 1-6.

13. Kwak JH, Lee J (2001) Enumeration of graph coverings, surface branched coverings and related group theory. Combinatorial and Computational Mathematics, pp: 97-161.

14. Kwak JH, Kim KH, Roush FW (2001) Combinatorial and Computational Mathematics: Present and Future. World Scientific, Singapore, pp: 97-161.

15. Pollington AD (1983) There is a long path in the divisor graph. Ars Combin 16: 303-304.

16. Pomerance $C$ (1983) On the longest simple path in the divisor graph. Congr Numer 40: 291-304. 